\title{
Sensitive and Noninvasive Detection of Aberrant SFRP2 and MGMT-B Methylation in Iranian Patients with Colon Polyps
}

\author{
M Alizade Naini ${ }^{1,3}$, P Mokarram ${ }^{3,2,4 *}$, S Kavousipour ${ }^{5}$, N Zare ${ }^{6}$, A Atapour ${ }^{5}$, M \\ Hassan Zarin ${ }^{1}$, G Mehrabani ${ }^{7}$, M Borji ${ }^{2}$
}

\begin{abstract}
Background: The pathogenesis of sporadic colorectal cancer (CRC) is influenced by the patient genetic background and environmental factors. Based on prior understanding, these are classified in two major pathways of genetic instability. Microsatellite instability (MSI) and CPG island methylator phenotype (CIMP) are categorized as features of the hypermethylated prototype, and chromosomal instability (CIN) is known to be indicative of the non-hypermethylated category. Secreted frizzled related protein 2 (SFRP2), APC1A in WNT signaling pathway and the DNA repair gene, O6-methylguanine-DNA methyltransferase (MGMT), are frequently hypermethylated in colorectal cancer. Detection of methylated DNA as a biomarker by easy and inexpensive methods might improve the quality of life of patients with CRC via early detection of cancer or a precancerous condition. Aim: To evaluate the rate of SFRP2 and MGMT hypermethylation in both polyp tissue and serum of patients in south Iran as compared with matched control normal population corresponding samples. Materials and Methods: Methylation-specific PCR was used to detect hypermethylation in DNA extracted from 48 polypoid tissue samples and 25 healthy individuals. Results: Of total polyp samples, $89.5 \%$ had at least one promoter gene hypermethylation. The most frequent methylated locus was SFRP2 followed by MGMT-B (81.2 and 66.6 percent respectively). Serologic detection of hypermethylation was $95 \%$ sensitive as compared with polyp tissue. No hypermethylation was detected in normal tissue and serum and its detection in patients with polyps, especially of serrated type, was specific. Conclusions: Serologic investigation for detection of MGMT-B, SFRP2 hypermethylation could facilitate prioritization of high risk patients for colonoscopic polyp detection and excision.
\end{abstract}

Keywords: Colorectal cancer - polyp - MGMT - SFRP2 - APC - methylation - serum diagnosis

Asian Pac J Cancer Prev, 17 (4), 2185-2193

\section{Introduction}

Colorectal cancer (CRC) is the third common cancer in the world with high mortality and morbidity. Although Iran is still a low risk country for CRC, but recent studies are in favour of rapid increasing in its incidence, especially in young people, approaching the western rates (Bishehsari et al., 2014). Remarkable increases in the sporadic CRC incidence are also seen in other Asian countries such as Japan, Singapore, Hong Kong, etc (Stoffel and Boland, 2015). Sporadic CRC which is known as a somatic genetic disease and epigenetic deregulation due to environmental hazards (Carethers and Jung, 2015). Other studies have also shown that distribution of colorectal cancer is strongly related to environmental factors (Hassanzade et al., 2011; Bishehsari et al., 2014). Unfortunately the burden of disease will increase dramatically in near future especially in young male $\&$ female population. The most important factor for this phenomena changes is dietary habits and lifestyle changes (Feldman and Friedman, 2010).

Based on previous molecular epidemiologic studies most sporadic CRCs possess normal-adenoma-cancer progression by initiating adenomas that transform into cancer over 1-2 decade. So generalized screening of proper age population with high quality strategic planning and good performance would be effective for reducing mortality and morbidities of CRCs. This can detect early stage cancers and pre-cancerous state such as inflammatory bowel disease (IBD) and adenoma. Desirable test for screening should also be simple, noninvasive, and acceptable for patients and physician with desirable with acceptable sensitivity and specificity. (Stracci et al., 2014; Leggett and Hewett, 2015)

Of available CRC screening methods; Fecal Occult Blood Test (FOBT) and Fecal Immunological Test (FIT) are two non-invasive methods with low sensitivity and specificity. Both have false (positive and negative) results, so should be substitute to more sensitive markers (Fisher

${ }^{1}$ Department of Internal Medicine, ${ }^{2}$ Department of Biochemistry, ${ }^{3}$ Gastroenterohepatology Research Center, Nemazi Hospital, School of Medicine, ${ }^{4}$ Colorectal Research Center, ${ }^{5}$ Ph.D Students in Biotechnology, School of Advanced Medical Science and Technologies, ${ }^{7}$ School of Medicine, Shiraz University of Medical Sciences, Shiraz, ${ }^{6}$ Department of Biochemistry, Science and Research Branch, Islamic Azad University, Fars, Iran*For correspondence: Mokaram2@gmail.com 
et al., 2006; Levin et al., 2008). Both double-contrast barium enema and virtual colonoscopy are sensitive, but are with important limitation due to radiation exposure and no chance of diagnostic and therapeutic resections (Feldman and Friedman, 2010) Proctosigmoidoscopy with direct visualization and the opportunity to perform biopsy and excision is a proper test for left colon evaluation. Colonoscopy is known as the gold standard method for detection of all colon lesions. On the other hand, even efficient colonoscopic evaluation with direct lesions detection, contract with its important limitations such invasiveness, specialist dependency, time-consuming and not being accepted by the patients.(Feldman and Friedman, 2010; Salehi et al., 2012). So it is mandatory to perform complementary simple, non-invasive, easily available and reliable methods to set priorities for high risk patients to be evaluated with colonoscopy.

Genetic alterations during initiation, transformation and progression of normal colonic stem cells into a precancerous state (adenomatous and serrated polyps), neoplastic, and metastatic cells is the outcome of complex and diverse pathogenesis with different molecular pathways.

The molecular pathogenesis and genetic alterations of sporadic CRC are significantly heterogeneous, but are classified in two major pathways of genetic instability. Of these, microsatellite instability (MSI) and CPG island methylator phenotype (CIMP) are categorized as hyper mutated prototype (16\% of sporadic CRCs), and chromosomal instability (CIN) is known as non-hyper methylated category ( $84 \%$ of sporadic CRCs). Hyper mutated group is the result of deficient DNA mismatch repair gene with high microsatellite instability (MSI) and BRAFV600E mutation. CIMP phenotype CRC is seen in nearly $20 \%$ with significant overlap with MSI CRCs and some non- hypermutated cancers. Non-hypermutated group is microsatellite stable, has proficient DNA with multiple somatic copy number alterations and aneuploidy in cancers with loss of heterozygosity of tumor suppressor genes such as APC and P53. These also contain oncogenic activation of KRAS and PIK3CA. Elevated microsatellite alteration at selected tetranucleotide is associated with metastatic behavior in both hyper and non-hyper mutated groups. CIMP positive tumors with aberrant hypermethylated CPG dinucleotides are poor prognostic and exhibit frequent KRAS and BRAF mutations (Okugawa et al., 2015).

Inactivated DNA mismatch repair (MMR) by hypermethylation or MLH1, MSH2, MSH6 gene mutation (MSI phenotype is represented in familial Lynch syndrome CRCs and sporadic type. Hyper methylated MLH1 and BRAF V6ooE mutation are more frequently in sporadic CRC. On the other hand, APC or B- cathenin gene (CTNNB1) alterations are not seen in sessile serrated polyp and traditional serrated adenoma but BRAF/KRAS mutations along with CIMP are frequently (Okugawa et al., 2015).

Detection of abnormal genetic pathways in abnormal tissue (cancer, polyp or chronic inflame mucosa), serum and stool are the base of new screening methods for early premalignant lesions and colorectal cancers (Summers et al., 2013). Molecular DNA studies and biomarker detection also not only could facilitate diagnostic approach for precancerous lesions, early detection of CRCs, and precancerous state can predict patient prognosis beyond staging and improve therapeutic decisions making with reducing metastatic risks or drug responsiveness (Okugawa et al., 2015). Not only gene mutations are known to be important in cancer formation, but also epigenetic alteration has been recognised as significant contributors. These alterations by aberrant DNA methylation, abnormal histone modification, and altered in non-coding RNAs expression are referred as different alterations, without changes in the DNA sequences (Okugawa et al., 2015).

Based on pathogenesis DNA, RNA, micro- RNA, epigenetic changes, protein and even antibody may be detected as biomarkers in body fluids (Summers et al., 2013; Takane et al., 2014) On the other hand, genetic and epigenetic alternations seen in serum are identical to those found in primary human cancers (Wang and Tang, 2008) Of these, detection of epigenetic changes especially DNA methylation is more simple and easier than genetic mutations (Takane et al., 2014). Many of the human genes particularly tumor suppressor genes (TSG) can be silenced by the hypermethylation of $\mathrm{CpG}$ islands (Herman and Baylin, 2003; Kim et al., 2010). Inactivation or loss of TSG products increases cell proliferation, which lead to malignant transformation (Herman and Baylin, 2003).

Transcription of various genes belonging to the BCathenin /WNT pathway (SFRP, MCC) signaling pathway and DNA mismatch repair gene (MLH1) are inactivated by methylation. In addition, down-regulation of WNT key genes such as APC1A; SFRP2 and Repair genes are prerequisite for the development of CRC (Coppedè et al., 2014).

Methylated DNA derived from early stage of cancer can be detected not only in tumor tissue as well as in plasma or serum (Lofton-Day et al., 2008; Herbst et al., 2011; De Maio et al., 2014; Wang et al., 2014).

Various studies show that there is a high level of circulating methylated DNA in peripheral blood of patients with malignancies including CRC in different population (Heitzer et al., 2015). SEPT9 methylation which is known as the first commercial DNA methylation test for the diagnosis of early colorectal cancer CRC was confirmed in nearly 8000 subjects with a specificity of approximately $90.0 \%$ (Heitzer et al., 2015) SDC2 as another novel, sensitive, and specific marker was identified for the detection of early-stage CRC(Tanzer et al., 2010; Oh et al., 2013). Moreover, other researchers identified blood-based molecular biomarkers including TMEFF2, NGFR (Kim et al., 2010; Herbst et al., 2011; Wang et al., 2014) and 12 genes (COL4A2, TSPYL5, TMEFF2, RASSF2, SPG20, EDIL3, CIDEB, ADAMTS1, EFHD1, STOX2, PPP1R3C, and UCHL1) (Takane et al., 2014) as useful screening marker in plasma samples with CRC. Interestingly, as compared to healthy population, methylated ALX4 and SEPT9 were reported more frequent in patients' peripheral blood with colorectal cancer and polyps (Oh et al., 2013). FBN2 and TCERG1L as cancerspecific genes with frequent methylation were seen in early stages of colorectal cancer during adenoma formation (Yi 
et al., 2012).

In other study, four genes, including BMP3, EYA2, ALX4, and vimentin were methylated in serum and stool of the majority of both colorectal cancers and premalignant adenomas (Zou et al., 2007). Furthermore, CA72-4, NEUROG1, TIMP-3+, TAC1, SEPT9 and EYA4 have been evaluated as biomarkers for adenoma and early CRC detection (Lofton-Day et al., 2008)

In spite of serum, other researchers reported the detection of SFRP2 and FBN1 in stool as a powerful screening method for CRC (Muller et al., 2004; Zhang et al., 2007) TFP12, GATA4, NIDRG4 and vimentin are suitable marker for cancer detection in stool and other body fluids determined by Lu et.al (Lu et al., 2014; Shah et al., 2014).

Up to date, no genetic defects have been reported in the MGMT gene in human cancer. However transcriptional silencing of this gene were seen in multiple tumor associated with hypermethylation of the $\mathrm{CpG}$ Island in its promoter zone (Shen et al., 2005). MGMT promoter methylation was detected with higher sensitivity in CSF specimens as compared to the serum for glioma patients. MGMT promoter methylation in CSF may provide a method for early diagnosis, treatment, monitoring of recurrence and prognosis of patients with glioma (Wang et al., 2015) Methylation of MGMT is also detected in gastric and colorectal cancers (Hibi et al., 2009) Furthermore, methylation of hMLH1, CDKN2A/p16, and MGMT revealed specific methylation profiles for tubular adenomas, tubulovillous/villous adenomas, and colorectal cancers (Psofaki et al., 2010).

MGMT promoter hypermethylation as an early event in human colorectal tumorigenesis and small colorectal adenoma is linked to the appearance of G to A mutations in the KRAS oncogene (Esteller et al., 1999). MGMT detection is a high sensitive marker in serum to predict colorectal cancer (Esteller et al., 1999). MGMT promoter has two loci, which we described as MGMT-A and MGMT-B. Based in our recent study (Mokarram et al., 2013) the methylation silencing of MGMT-B rather than MGMT-A was significantly associated with KRAS gene mutation in CRC patients but not in normal tissues. In another study, recently it was demonstrated that hypermethylation of the MGMT-B and the SFRP2 gene promoter regions might be involved in IBD development and methylation of MGMT-B in IBD patients. So its detection may provide a method for early production of IBD-associated neoplasia (Mokarram et al., 2015).

Based on these results, this is hypothesized that methylation of MGMT-B might be responsible for dysregulation of signaling through KRAS mutation in transforming to polyp lesions progressing to malignant mass. It is well established that the WNT signalling pathway involving b-catenin has a crucial role in the development of colorectal carcinomas through the conventional adenoma-carcinoma sequence (Murakami et al., 2015). Differences in APC expression caused by promoter methylation are also selected to WNT signalling as an early event in colorectal tumors and early polyp lesions (Segditsas et al., 2008). SFRP2 methylation may also reflect the malignant potential of adenomas.
Thus, patients with faecal hyper methylated SFRP2 may belong to high-risk individuals and need more sensitive and precise clinical examinations and follow-up (Huang et al., 2007).

Serum SFRP2 methylation status also represents as a non-invasive marker for CRC detection and staging and hypermethylated SFRP2 may have prognostic relevance in patients with CRC (Tang et al., 2011).

SFRP2 methylation in faecal DNA increases significantly from healthy controls to patients with hyperplastic polyps and to patients with adenomas. SFRP2 methylation may serve as a marker for molecular stool-based adenoma and CRC screening (Oberwalder et al., 2008).

Moreover, ITGA4, SFRP2 and p16 promoter methylation in stool samples indicates high sensitivity and specificity for the detection of epigenetic abnormalities in patients with colorectal adenomas and cancers (Chang et al., 2010). On the other hand combination of GATA5 and SFRP2 methylation could be promising as a marker for the detection and diagnosis of CRC and adenomas (Zhang et al., 2007). DNA methylation of p14ARF, RASSF1A and APC1A genes, defines a poor prognosis subset of CRC patients independently of both tumor stage and differentiation (Zhang et al., 2007).

Therefore, different studies suggest that downregulation of Wnt key genes such as APC1a; SFRP2 and repair genes are prerequisite for the development of CRC. In this study for the first time, early alteration of APC1A, SFRP2 and MGMT-B genes methylation were evaluated in patients' polyp tissues, serum as compared with normal matched control tissues. These epigenetic alterations may have probable potential reliable clinical biomarkers for diagnostic, prognostic, and therapeutic applications of polyp-CRCs transformation.

\section{Materials and Methods}

\section{Patients and sample (polyp, normal mucosa and serum specimens)}

During 2 years (2011-2013), all patients who referred to clinical centers affiliated to Shiraz University of Medical Sciences and had polyp lesions in colonoscopic evaluation, were reevaluated by one gastroenterologist to include patients with new onset polyp, not previously treated with negative family history of CRCs or IBD to be included in this study. Of 54 patients' polyp tissues 48 polyp samples as well as corresponding normal tissues were included in this research. Corresponding serum of this patients were also obtained, but due to limited availability of kit, only 25 patients 'serum samples were evaluated. Serum and mucosal tissue biopsy of 25 matched healthy volunteer with normal colonoscopic evaluation were included as control group. The patients were grouped based on patients 'age, $(\geq 35$ and $<53)$, location (proximal and distal or right and left) and histologic changes (sessile serrated/ adenoma polyp with hyperplasia, traditional serrated adenoma and adenoma with serrated architecture) of polyps. All enrolled individuals provided written informed consent. Ethics committee and institutional review board of Shiraz University of Medical Sciences approved the study. Fresh 
samples including tissue and serum were snap-frozen and stored at $-70^{\circ} \mathrm{C}$ until processing.

\section{Extraction of DNA and Methylation specific PCR}

Genomic DNA was extracted from samples, as described in previous research, we used the standard phenol/chloroform method for DNA extraction from fresh polyp samples (Mokarram et al., 2008).

We determined promoter methylation status of MGMT, SFRP2 and APC1 A genes by chemical treatment with sodium bisulfite and subsequent MSP, as described in our previous research (Mokarram et al., 2008). In brief, this technique uses bisulfate modification to convert unmethylated, but not methylated cytosine to uracil. MSP utilizes this difference to amplify specifically either methylated or unmethylated DNA.

25 serum from healthy participants as well as 25 serum from polyp patient were selected for direct methylation assay by zymoresearch kit (EZ DNA MethylationDirect $^{\mathrm{TM}}$ Kit D5020).

The hot-start methylation specific PCR(MS-PCR) reactions were performed in a $50 \mu \mathrm{L}$ reaction volume containing 25 pmol of sense and antisense primers, 0.2 $\mathrm{mmol} / \mathrm{L}$ dNTPs, and $80 \mathrm{ng}$ bisulfite-modified DNA in $1 \mathrm{X}$ PCR buffer provided by Taq enzyme supplier. The reaction mixture was denatured at $95^{\circ} \mathrm{C}$ for $5 \mathrm{~min}$, after which $1.5 \mathrm{U}$ Taq polymerase was added; then amplified by 40 cycles, each consisting of $30 \mathrm{~s}$ denaturation at $95^{\circ} \mathrm{C}$, proper annealing temperature for each gene (Table 1) and $30 \mathrm{~s}$ polymerization at $72^{\circ} \mathrm{C}$, followed by a single 10 -min extension at $72^{\circ} \mathrm{C}$.

Locus specific PCR primers for three genes MGMT-B, SFRP2,APC1A were specifically designed for methylation specific PCR and located at each gene promoter region. The sequences, annealing temperature of each primer used for amplification and PCR products size were described in Table 1 .

The universal methylated DNA (chemicon) was used as positive control for methylated alleles of MGMT-B, SFRP2 and APC1A and DNA from normal lymphocytes was used as the negative control. $10 \mu \mathrm{L}$ of amplified
PCR products were mixed with $5 \mu \mathrm{L}$ of loading dye and electrophoresed on $2.5 \%$ agarose gel containing gel red with TBE buffer and visualized under UV illumination.

\section{Statistical analysis}

As compared to DNA methylation assay of polyp tissues (as a gold standard) the sensitivity and specificity of the serum DNA assays were calculated (with $95 \%$ confidence interval (CI)). To compare the characteristics of the different groups of patients, $\chi 2$ tests and Fisher's exact tests were used. All statistical tests were performed using SPSS version 11.0 software (SPSS, Inc., Chicago, IL, USA). All values were two-sided and $\mathrm{P}<0.05$ was considered to indicate a statistically significant difference.

\section{Results}

\section{Distribution of selected polyp patients}

Of total 54 patients with colonoscopic polyp detection, 48 had criteria to be included in this research; of these, 17, 28 and 3 were sessile serrated/ adenoma polyp with hyperplasia, traditional serrated adenoma and adenoma with serrated architecture, respectively. Twenty polyps were located in proximal and 26 in distal part of colon. Based on the median age of patient's population (53 years), 23 and 25 of 48 patients were respectively in $>53$ and $\leq 53$ year old groups.

\section{Prevalence of MGMT-B, APC1a and SFRP2}

Polyp tissues of 48 patients were analyzed for methylation of SFRP2, APC1A and MGMT-B. Clinicobiological characteristics of total patients are presented in

Table 2. Distribution of Selected Characteristic of Cases

\begin{tabular}{lc}
\hline Variable & $\mathrm{N}(\%)$ \\
\hline Gender & \\
Male $(\%)$ & 0.353 \\
Female(\%) & 0.647 \\
Median age & 53 \\
Mean age & $60 \pm 18$ \\
\hline
\end{tabular}

Table 1. Primers used for Methylation Specific PCR (MSP)

\begin{tabular}{|c|c|c|c|}
\hline \multicolumn{2}{|c|}{ Gene } & \multirow{2}{*}{$\begin{array}{l}\text { Primer sequence }(5 \rightarrow 3) \\
\text { TGCGTGTTTTTATTTTCGTAGTTCGC }\end{array}$} & \multirow{2}{*}{$\frac{\text { Annealing Temperature }\left({ }^{\circ} \mathrm{C}\right)}{59}$} \\
\hline SFRP2 & MF & & \\
\hline & MR & CCCTAAATACCGCCGCTCGCCCG & \\
\hline & $\mathrm{UF}$ & GTTTTGTGTGTTTTTTATTTTTGTAGTTTGT & \\
\hline & UR & TCCССТАAАТАCCACCACTCACCCA & \\
\hline \multirow[t]{4}{*}{ APC1A } & MF & TATTGCGGAGTGCGGGT & 63 \\
\hline & MR & TCGACGAACTCCCGACG & \\
\hline & $\mathrm{UF}$ & GTGTTTTATTGTGGAGTGTGGGT & \\
\hline & UR & ССААТСААСАААСТСССААСА & \\
\hline \multirow[t]{4}{*}{ MGMT-B } & MF & GGTCGTTTGTACGTTCGC & 59 \\
\hline & MR & TAACCCTTCGACCGATACAA & \\
\hline & UF & GTAGGTTGTTTGTATGTTTGT & \\
\hline & UR & TAACCCTTCAACCAATACAAACC & \\
\hline
\end{tabular}


Table 3. Stratification Analysis of Cases and Genes Promoter Methylation Frequencies

\begin{tabular}{|c|c|c|c|c|c|}
\hline \multirow{2}{*}{\multicolumn{2}{|c|}{ Variable }} & \multicolumn{3}{|c|}{ Positive Methylation N (\%) } & \\
\hline & & \multirow{2}{*}{$\begin{array}{r}\text { SFRP2 } \\
0.823\end{array}$} & \multirow{2}{*}{$\frac{\text { MGMT }}{0.895}$} & \multirow{2}{*}{$\frac{\mathrm{APC} 1 \mathrm{~A}}{0.758}$} & \multirow{2}{*}{$\frac{\text { At least one methylated gene }(\%)}{0.75}$} \\
\hline Sex & Male & & & & \\
\hline & Female & 0.811 & 0.895 & 0.727 & 0.72 \\
\hline & Total & 0.812 & 0.895 & 0.71 & 0.895 \\
\hline & $\mathrm{P}$ & 1 & 1 & 1 & 0.325 \\
\hline \multicolumn{6}{|l|}{ Age } \\
\hline & $<53$ & 0.826 & 0.756 & 0.667 & 0.708 \\
\hline & $\geq 53$ & 0.88 & 0.8 & 0.64 & 0.92 \\
\hline & $\mathrm{P}$ & 1 & 1 & 1 & 0.139 \\
\hline \multicolumn{6}{|c|}{ Dysplastic serrated polyp } \\
\hline & sessile serrated Adenoma/ polyp with hyperplasia & 0.588 & 0.352 & 0.294 & 0.4 \\
\hline & Traditional serrated adenoma & 0.964 & 0.892 & 0.857 & 0.92 \\
\hline & Adenoma with serrated architecture & 0.333 & 0.333 & 33.3 & 0.2 \\
\hline & $\mathrm{P}$ & 0.001 & 0.006 & 0.005 & 0.001 \\
\hline \multicolumn{6}{|c|}{ Location } \\
\hline & Proximal & 0.863 & 0.81 & 0.77 & 0.67 \\
\hline & Distal & 0.77 & 0.538 & 0.653 & 0.795 \\
\hline & $\mathrm{P}$ & 1 & 0.5 & 1 & 1 \\
\hline
\end{tabular}

Table 4. Correlation of MGMT and SFRP2 methylation in tissue and serum

\begin{tabular}{lcc}
\hline Variables & $\mathrm{N}(\%)$ of methylation & Pearson correlation \\
\hline (Serum) & & MGMT \\
MGMT & 0.818 & $\mathrm{R}=0.531$ \\
SFRP2 & 0.87 & $\mathrm{P}=0.004$ \\
(Tissue) & & $\mathrm{SFRP} 2$ \\
MGMT & 0.666 & $\mathrm{R}=0.8$ \\
SFRP2 & 81.2 & $\mathrm{P}=0.0001$ \\
\hline
\end{tabular}

Table 5. The Comparison of Sensitivity and Specificity of MGMT and SFRP2 Methylation in Serum

\begin{tabular}{lcc}
\hline & MGMT (\%) & SFRP2(\%) \\
\hline Sensitivity & 0.95 & 0.9 \\
Specificity & 100 & 100 \\
Predective Positive Value (PPV) & 100 & 100 \\
Predective Negative Value (PNV) & 0.95 & 0.93 \\
\hline
\end{tabular}

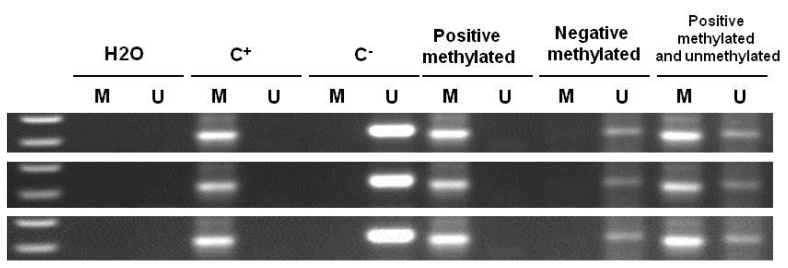

Figure 1. Representative examples of MSP reactions for promoter methylation analysis of SFRP2 gene in polyp samples. The presence of a visible PCR product in those lanes marked $U$ indicates the presence of unmethylated genes; the presence of a product in those lanes marked $M$ indicates the presence of methylated genes. Lane 1 indicates the 50 bp DNA size marker. Universal methylated DNA (UMD), unmethylated lymphocytes (lymphocytes) DNA and $\mathrm{H} 2 \mathrm{O}$ were used as positive, negative controls and NTC, respectively

Table 2. The frequencies of the methylation status of the three promoters and their relationship with the clinical characteristics of the patients were summarized in Table 3.
Several studies have reported age-dependent methylation (Mokarram, et al., 2008), therefore, we divided both polyp and control groups into $\geq 53$ and < 53 year old groups. The median age of polyp patients (53 years) was chosen for this division. Although more methylation was seen in older patients but it was not statistically significant. Therefore, neither gender nor age was associated with the promoter methylation status of the genes. However, we had more gene methylation in traditional serrated polyp and equivalent sensitivity of gene methylation in proximal and distal location. No methylation of SFRP2, APC1A and MGMT-B were detected in patients' corresponding adjacent normal mucosal tissues or in normal volunteers' tissues /serum.

The most frequent methylated locus was SFRP2 ( 81.2 $\% ; 39$ of 48$)$, followed by APC1A $(71 \% ; 34$ of 48$)$ and MGMT $(66.6 \% ; 32$ of 48). The frequency of promoter methylation in polyp patients with at least one of 3 genes methylated was $43 / 48(89.5 \%) .41$ of $48(85.4 \%)$ of polyp tissue had MGMT-B or SFRP2 hypermethylation and 43 of $48(89.5 \%)$ patients had methylation of APC1A or SFRP2 while 35 of 48 (72.9\%) had MGMT-B or APC1A promoter hypermethylation.

The frequency of methylation for both SFRP2 and APC1A, MGMT and SFRP2, MGMT and APC1A methylation were 39/48 (\% 81.5), 29/48 (\% 60), 15/48 (\%31), respectively. Twenty four polyp samples had simultaneous $\mathrm{CpG}$ island hypermethylation of all three genes. These samples were more in traditional serrated adenoma category.

MGMT and SFRP2 methylation analysis was performed in 25 plasma samples using MS-PCR. MGMT and SFRP2 methylation assay were respectively positive in 22 and 23 serum samples per methylation test. 18 out of $22(81.8 \%)$ and 20 out of 23 samples were methylated for MGMT and SFRP2, respectively. Regarding to Table 3 , these two genes were selected for serum evaluation due to be more methylated in 48 polyp tissue samples and we did not perform further study on APC1a methylation in serum. According to Table 4, we could obtain the 
significant correlation between methylation status in serum and polyp tissue without any methylation in serum of healthy control. Statistical analysis revealed a significant correlation between the frequency of MGMT and SFRP2 methylation in serum and tissue of patients with polyps. ( $\mathrm{p}=.004, \mathrm{p}=0.001$, Table 4 ). As compared to polyp tissues methylation, the sensitivity and specificity of the serum DNA methylated assays were calculated. MGMT and SFRP2 methylation demonstrated approximately $95 \%$ and $100 \%$, respectively (Table 5). In addition, some examples of SFRP2 methylation are shown in Figure 1.

\section{Discussion}

Colorectal cancer screening could reduce the incidence and mortality of CRC, based on relative invasiveness, being time and resource consuming and specialist dependency, colonoscopy as a gold standard method for abnormal lesion detection, is not an acceptable efficient procedure of choice for CRC screening. On the other hand, cells of colon cancer and polypoid lesions are continuously shed into the serum and stools and their genetic and epigenetic alterations could be detected by molecular tests for altered molecular DNA methylations. This has been proposed as achievable screening methods for the early detection of cancerous and precancerous lesions (Zhang et al., 2015).

Currently, an increasing number of hypermethylated genes in biological samples such as serum and stool have been reported as potential biomarkers for the detection of colorectal neoplasia (Lu et al., 2014; Zhang et al., 2015)

In the present study, the feasibility of detecting methylated DNA in serum samples was evaluated as a noninvasive screening tool for the serum-based detection and/or monitoring of polyp-CRC patients. Our study demonstrates that hypermethylation of the APC1A and the SFRP2 gene promoter region as genes involving in the WNT signaling or MGMT-B and SFRP2 methylation are associated in polyp developing lesions.

All three methylation markers of the genes selected for this study have been identified in CRC previously. Functional loss of negative WNT regulators by epigenetic gene silencing, through both DNA methylation and histone modification of TSG-associated promoters, has been frequently reported to contribute to the activation or amplification of aberrant WNT/ $\beta$-catenin signalling in tumors. This phenomenon has crucial role in the development of colorectal carcinomas through the conventional adenoma-carcinoma sequence (Murakami et al., 2015). Down regulation of extracellular WNT antagonists such as SFRP1, SFRP2, SFRP4, SFRP5, WIF1, DKK1, DKK2 and DKK3 due to abnormal promoter methylation correlates with constitutive activation of canonical WNT/ $\beta$-catenin signalling in various tumors. Epigenetic silencing of APC and AXIN2 through promoter methylation underlies an alternative mechanism to genetic alteration for the loss of these genes' function in colon cancer development (Naghibalhossaini et al., 2012).The occurrence of such epigenetic inactivation would predispose the stem/or progenitor cells to abnormal clonal expansion, without cell differentiation and maturation. These cells then become effectively addicted to the over-activity of WNT signalling, and later acquire further inactivating or activating mutations in downstream signalling factors (such as APC or CTNNB1) of this pathway. The accumulation of multiple further epigenetic alterations can also subsequently act in a complementary manner to give genetic mutations full capacity to up regulate WNT signalling and facilitate tumor progression (Ying and Tao, 2009).

A recent study has shown that only promoter $1 \mathrm{~A}$ is affected in CRC progression and promoter 1B seems resistant to methylation in multiple cancer types (Segditsas et al., 2008). Our data also confirmed the hypermethylation of APC1A with less frequency in polyp samples.

Recently, researchers showed that non polypoid adenomas display less APC mutations compared to polypoid adenomas. APC silencing by promoter hypermethylation occurred at similar frequencies in both phenotypes. On the other hand, other upstream WNT signal regulating genes may present an alternative mechanism of constitutive WNT-pathway activation in CRC. Methylation of the WNT-antagonists may provide an alternative mechanism of WNT-pathway activation next to APC methylation (Voorham et al., 2013).

Furthermore, restoring SFRP expression in colorectal cancer cells can up regulate autocrine or paracrine WNT signaling. Given the crosstalk and overlap between molecular pathways, especially in cell signaling, it is entirely expected that WNT activation in colorectal tumors will be influenced by epigenetic changes in genes that act primarily in other pathways (Segditsas and Tomlinson, 2006).

On the other hand, genetics and epigenetics alterations have been found to be characteristic of malignancy and hence can be used as targets for detection of neoplasms in serum. In this regard, RASSF1A hypermethylation in tissues and serum and its protein expression may be a valid, reliable and sensitive tool for detection and follow up of patients with breast cancer (Hagrass et al., 2014). The presence of methylated TFPI2 DNA in serum is strongly associated with metastatic melanoma disease (Lo Nigro et al., 2013). In addition, a significant inverse association between tumor percent LINE-1 methylation and tumor stage/grade has been reported (van Bemmel et al., 2012).

In CRC, epigenetic silencing has been observed not only for regulators of Wnt signalling upstream and downstream in the pathway, such as the extracellular Wnt inhibitors SFRP1, SFRP2,SFRP3, SFRP4, SFRP5, WIF1, DKK1, DKK3, DACT3 and APC, AXIN2, CDH1, SOX17, respectively to progress from adenoma to carcinoma (Silva et al., 2014). Hypermethylated SFRPs was detectable not only in stools of patients with CRC, but also in stools of patients with premalignant lesions, such as advanced adenomas, suggesting that hypermethylated SFRP is a sensitive fecal-based molecular marker for screening CRC and premalignant lesions. These patients need to be prioritized for clinical examinations, diagnostic and therapeutic resections of lesions and follow-up (Wang and Tang, 2008).

Previous studies also revealed the sensitivity and specificity of the methylated SFRP2 genes in tissue and 
stool of patients with colorectal cancer and adenoma (Takeda et al., 2011) (Takeda et al., 2011). Our data also showed that hypermethylation of SFRP2 is able to use as sensitive marker in serum of polyp lesions. Therefore, hypermethylation of SFRP2 could introduce as specific marker in precancerous lesion of polyp patients in serum. In addition, only one study showed that the methylation of SFRP2 could be a specific marker in serum of patients with hyperplastic polyp and colorectal cancer (Tang et al., 2011) that the percentage in our study was higher than their reports.

To the best of our knowledge, due to high level of methylation for SFRP2 in our samples, methylated SFRP2 could be one of the most sensitive marker in precancerous lesions. In addition, this gene with high sensitivity and specificity $(95 \%, 100$ respectively) could be a promising non-invasive marker in serum for polyp-CRCs detection. Our data is consistent with others that showed methylated SFRP2 is a diagnostic marker in fecal DNA for CRC detection with $80 \%$ sensitivity and $77 \%$ specificity (Okugawa et al., 2015).

Gene methylation status of SFRP2 and APC1a in our polyp tissues was not associated with the polyp location. Due to lack of information, the correlation for these methylation in polyps in different regions of colon should be further investigated in larger population.

To date, no published studies have reported an association between genetic defects in the MGMT gene, such as mutations and/or deletions, and human cancer. However, several studies have reported that transcriptional silencing of this gene through hypermethylation of the $\mathrm{CpG}$ Island in its promoter to be associated with the appearance of G-to-A point mutations in the KRAS, P53 genes during colorectal tumorigenesis especially in the MSI-Subset of colorectal cancers and in precursor lesions (Whitehall et al., 2001; Halford et al., 2005). In addition, to date a large number of hypermethylated genes such as APC1a, SFRP2 and MGMT have been found in methylation assay for early detection of CRC (Okugawa et al., 2015).

In previous study, higher frequency of MGMT-B methylation was seen in patients with CRCs (Mokarram et al., 2013). This phenomenon was accompanied with a good correlation between MGMT methylation and increasing risk of KRAS and P53 mutation (Halford et al., 2005). In this study MGMT methylation was evaluated in serum and tissues of patients with poly; as a known precancerous state. Therefore, besides SFRP2, MGMT hyper methylation in serum and tissue of patients with polyp could be the second marker for our population which is consistent with Lee et al research results (Lee et al., 2009)

Methylation of MGMT and SFRP2 genes were strongly associated with type of polyp lesions. Furthermore, based on binary logistic test $(\mathrm{OR}=1.4, \% 95 \mathrm{CI}(2-10)$, a trend in the higher methylation were seen in older patients $(>53 \mathrm{Y})$ and significant increase were detected in traditional serrated adenoma $(\mathrm{OR}=2.5,95 \% \mathrm{CI},(0.1-4)$. This convinces the hypothesis of increasing cancer incidence in older patients and traditional polyps. This finding was also consistent with the Takada et al hypothesis (Takeda et al., 2011) that accumulation of MGMT and SFRP2 methylation could lead the traditional polyp lesion to cancer.

There was no correlation between MGMT-B methylation and polyp localization. This result contract with Kycler study which showed no correlations between methylation status of MGMT and clinical features like age, gender, and polyp localization (Kycler et al., 2012).

In conclusion, MGMT-B promoter methylation accompanied with SFRP2 and APC1A has also been detected in the south Iranian patients with polyp but not in normal population. Aberrant methylated gene may be detected in polyp tissues and serum concomitantly, so there is a potential capacity for serum to be used for early detection of polyps as a precancerous state. Serum detection for methylation status was sensitive and specific, so it could be used in a qualified screening planning by prioritize patients for colonoscopy and waiting list deduction. To improve the beneficial effects, cohort studies with larger sample size are recommended.

\section{Acknowledgements}

This study was supported by Grant Number 4761 from Vice-chancellor for Research Affairs of Shiraz University of Medical Sciences.

\section{References}

Bishehsari F, Mahdavinia M, Vacca M, et al (2014). Epidemiological transition of colorectal cancer in developing countries: Environmental factors, molecular pathways, and opportunities for prevention. World J Gastroenterol, 20, 6055-72.

Carethers JM, Jung BH (2015). Genetics and Genetic Biomarkers in Sporadic Colorectal Cancer. Gastroenterol, 149, 117790.e3.

Chang E, Park DI, Kim YJ, et al (2010). Detection of colorectal neoplasm using promoter methylation of ITGA4, SFRP2, and p16 in stool samples: a preliminary report in Korean patients. Hepatogastroenterol, 57, 720-7.

Coppedè F, Lopomo A, Spisni R, et al (2014). Genetic and epigenetic biomarkers for diagnosis, prognosis and treatment of colorectal cancer. World J Gastroenterol, 20, 943-56.

De Maio G, Rengucci C, Zoli W, et al (2014). Circulating and stool nucleic acid analysis for colorectal cancer diagnosis. World J Gastroenterol, 20, 957-67.

Esteller M, Hamilton SR, Burger PC, et al (1999). Inactivation of the DNA repair gene O6-methylguanine-DNA methyltransferase by promoter hypermethylation is a common event in primary human neoplasia. Cancer Res, 59, 793-7.

Feldman M, Friedman LS (2010). Sleisenger and Fordtran's Gastrointestinal and Liver Disease. In Eds Saunders elsevier. Philadelphia.

Fisher JA, Fikry C, Troxel AB (2006). Cutting cost and increasing access to colorectal cancer screening: another approach to following the guidelines. Cancer Epidemiol Biomarkers Prev, 15, 108-13.

Hagrass HA, Pasha HF, Shaheen MA, et al (2014). Methylation status and protein expression of RASSF1A in breast cancer patients. Mol Biol Rep, 41, 57-65.

Halford S, Rowan A, Sawyer E, et al (2005). O(6)-methylguanine methyltransferase in colorectal cancers: detection of 
mutations, loss of expression, and weak association with G:C >A:T transitions. Gut, 54, 797-802.

Hassanzade J, Molavi EVH, Farahmand M, et al (2011). Incidence and mortality rate of common gastrointestinal cancers in south of Iran, a population based study. Iran $J$ Cancer Prev, 4, 163-9.

Heitzer E, Ulz P, Geigl JB (2015). Circulating tumor DNA as a liquid biopsy for cancer. Clin Chem, 61, 112-23.

Herbst A, Rahmig K, Stieber P, et al (2011). Methylation of NEUROG1 in serum is a sensitive marker for the detection of early colorectal cancer. Am J Gastroenterol, 106, 1110-8.

Herman JG, Baylin SB (2003). Gene silencing in cancer in association with promoter hypermethylation. $N$ Engl J Med, 349, 2042-54.

Hibi K, Goto T, Mizukami H, et al (2009). MGMT gene is aberrantly methylated from the early stages of colorectal cancers. Hepatogastroenterol, 56, 1642-4.

Huang ZH, Li LH, Yang F, et al (2007). Detection of aberrant methylation in fecal DNA as a molecular screening tool for colorectal cancer and precancerous lesions. World $J$ Gastroenterol, 13, 950-4.

Kim MS, Lee J, Sidransky D (2010). DNA methylation markers in colorectal cancer. Cancer Metastasis Rev, 29, 181-206.

Kycler W, Szarzynska B, Lozinski C, et al (2012). Analysis of O6-methylguanine-DNA methyltransferase methylation status in sporadic colon polyps. Rep Pract Oncol Radiother, 17, 13-8.

Lee BB, Lee EJ, Jung EH, et al (2009). Aberrant methylation of APC, MGMT, RASSF2A, and Wif-1 genes in plasma as a biomarker for early detection of colorectal cancer. Clin Cancer Res, 15, 6185-91.

Leggett BA, Hewett DG (2015). Colorectal cancer screening. Intern Med J, 45, 6-15.

Levin B, Lieberman DA, McFarland B, et al (2008). Screening and surveillance for the early detection of colorectal cancer and adenomatous polyps, 2008: a joint guideline from the American Cancer Society, the US Multi-Society Task Force on Colorectal Cancer, and the American College of Radiology. CA Cancer J Clin, 58, 130-60.

Lo Nigro C, Wang H, McHugh A, et al (2013). Methylated tissue factor pathway inhibitor 2 (TFPI2) DNA in serum is a biomarker of metastatic melanoma. J Invest Dermatol, 133, 1278-85.

Lofton-Day C, Model F, Devos T, et al (2008). DNA methylation biomarkers for blood-based colorectal cancer screening. Clin Chem, 54, 414-23.

Lu H, Huang S, Zhang X, et al (2014). DNA methylation analysis of SFRP2, GATA4/5, NDRG4 and VIM for the detection of colorectal cancer in fecal DNA. Oncol Lett, 8, 1751-6.

Mokarram P, Kavousipour S, Sarabi MM, et al (2015). MGMT-B gene promoter hypermethylation in patients with inflammatory bowel disease - a novel finding. Asian Pac J Cancer Prev, 16, 1945-52.

Mokarram P, Naghibalhossaini F, Saberi Firoozi M, et al (2008). Methylenetetrahydrofolate reductase C677T genotype affects promoter methylation of tumor-specific genes in sporadic colorectal cancer through an interaction with folate/ vitamin B12 status. World J Gastroenterol, 14, 3662-71.

Mokarram P, Zamani M, Kavousipour S, et al (2013). Different patterns of DNA methylation of the two distinct O6methylguanine-DNA methyltransferase (O6-MGMT) promoter regions in colorectal cancer. Mol Biol Rep, 40, 3851-7.

Muller HM, Oberwalder M, Fiegl H, et al (2004). Methylation changes in faecal DNA: a marker for colorectal cancer screening? Lancet, 363, 1283-5.

Murakami T, Mitomi H, Saito T, et al (2015). Distinct WNT/ beta-catenin signaling activation in the serrated neoplasia pathway and the adenoma-carcinoma sequence of the colorectum. Mod Pathol, 28, 146-58.

Naghibalhossaini F, Zamani M, Mokarram P, et al (2012). Epigenetic and genetic analysis of WNT signaling pathway in sporadic colorectal cancer patients from Iran. Mol Biol Rep, 39, 6171-8.

Oberwalder M, Zitt M, Wontner C, et al (2008). SFRP2 methylation in fecal DNA--a marker for colorectal polyps. Int J Colorectal Dis, 23, 15-9.

Oh T, Kim N, Moon Y, et al (2013). Genome-wide identification and validation of a novel methylation biomarker, SDC2, for blood-based detection of colorectal cancer. J Mol Diagn, 15, 498-507.

Okugawa Y, Grady WM, Goel A (2015). Epigenetic Alterations in Colorectal Cancer: Emerging\&\#xa0;Biomarkers. Gastroenterol, 149, 1204-25.

Psofaki V, Kalogera C, Tzambouras N, et al (2010). Promoter methylation status of hMLH1, MGMT, and CDKN2A/p16 in colorectal adenomas. World J Gastroenterol, 16, 3553-60.

Salehi R, Mohammadi M,Emami MH, et al (2012). Methylation pattern of SFRP1 promoter in stool sample is a potential marker for early detection of colorectal cancer. Adv Biomed Res, $1,87$.

Segditsas S, Sieber OM, Rowan A, et al (2008). Promoter hypermethylation leads to decreased APC mRNA expression in familial polyposis and sporadic colorectal tumours, but does not substitute for truncating mutations. Exp Mol Pathol, 85, 201-6.

Segditsas S, Tomlinson I (2006). Colorectal cancer and genetic alterations in the Wnt pathway. Oncogene, 25, 7531-7.

Shah R, Jones E, Vidart V, et al (2014). Biomarkers for early detection of colorectal cancer and polyps: systematic review. Cancer Epidemiol Biomarkers Prev, 23, 1712-28.

Shen L, Kondo Y, Rosner GL, et al (2005). MGMT promoter methylation and field defect in sporadic colorectal cancer. J Natl Cancer Inst, 97, 1330-8.

Silva AL, Dawson SN, Arends MJ, et al (2014). Boosting Wnt activity during colorectal cancer progression through selective hypermethylation of Wnt signaling antagonists. BMC Cancer, 14, 891 .

Stoffel EM, Boland CR (2015). Genetics and Genetic Testing in Hereditary Colorectal Cancer. Gastroenterol, 149, 1191-203.

Stracci F, Zorzi M, Grazzini G (2014). Colorectal Cancer Screening: Tests, Strategies, and Perspectives. Frontiers Public Health, 2, 210.

Summers T, Langan RC, Nissan A, et al (2013). Serum-based DNA methylation biomarkers in colorectal cancer: potential for screening and early detection. J Cancer, 4, 210-6.

Takane K, Midorikawa Y, Yagi K, et al (2014). Aberrant promoter methylation of PPP1R3C and EFHD1 in plasma of colorectal cancer patients. Cancer Med, 3, 1235-45.

Takeda M, Nagasaka T, Dong-Sheng S, et al (2011). Expansion of $\mathrm{CpG}$ methylation in the SFRP2 promoter region during colorectal tumorigenesis. Acta Med Okayama, 65, 169-77.

Tang D, Liu J, Wang DR, et al (2011). Diagnostic and prognostic value of the methylation status of secreted frizzled-related protein 2 in colorectal cancer. Clin Invest Med, 34, 88-95.

Tanzer M, Balluff B, Distler J, et al (2010). Performance of epigenetic markers SEPT9 and ALX4 in plasma for detection of colorectal precancerous lesions. PLoS One, 5, 9061.

van Bemmel D, Lenz P, Liao LM, et al (2012). Correlation of LINE-1 methylation levels in patient-matched buffy coat, serum, buccal cell, and bladder tumor tissue DNA samples. Cancer Epidemiol Biomarkers Prev, 21, 1143-8.

Voorham QJ, Janssen J, Tijssen M, et al (2013). Promoter methylation of Wnt-antagonists in polypoid and nonpolypoid 

colorectal adenomas. BMC Cancer, 13, 603 .

Wang D-R, Tang D (2008). Hypermethylated SFRP2 gene in fecal DNA is a high potential biomarker for colorectal cancer noninvasive screening. World J Gastroenterol, 14, 524-31.

Wang X, Kuang YY, Hu XT (2014). Advances in epigenetic biomarker research in colorectal cancer. World $J$ Gastroenterol, 20, 4276-87.

Wang Z, Jiang W, Wang Y, et al (2015). promoter methylation in serum and cerebrospinal fluid as a tumor-specific biomarker of glioma. Biomed Rep, 3, 543-8.

Whitehall VL, Walsh MD, Young J, et al (2001). Methylation of O-6-methylguanine DNA methyltransferase characterizes a subset of colorectal cancer with low-level DNA microsatellite instability. Cancer Res, 61, 827-30.

Yi JM, Dhir M, Guzzetta AA, et al (2012). DNA methylation biomarker candidates for early detection of colon cancer. Tumour Biol, 33, 363-72.

Ying Y, Tao Q (2009). Epigenetic disruption of the WNT/betacatenin signaling pathway in human cancers. Epigenetics, 4, 307-12.

Zhang W, Bauer M, Croner RS, et al (2007). DNA stool test for colorectal cancer: hypermethylation of the secreted frizzledrelated protein-1 gene. Dis Colon Rectum, 50, 1618-26.

Zhang X, Song YF, Lu HN, et al (2015). Combined detection of plasma GATA5 and SFRP2 methylation is a valid noninvasive biomarker for colorectal cancer and adenomas. World J Gastroenterol, 21, 2629-37.

Zou H, Harrington JJ, Shire AM, et al (2007). Highly methylated genes in colorectal neoplasia: implications for screening. Cancer Epidemiol Biomarkers Prev, 16, 2686-96. 